\title{
Near-infrared spectroscopy of SN 2009ip's 2012 brightening reveals a dusty pre-supernova environment
}

\author{
Nathan Smith, ${ }^{1 \star}$ Jon C. Mauerhan, ${ }^{1}$ Mansi M. Kasliwal ${ }^{2}$ and Adam J. Burgasser ${ }^{3}$ \\ ${ }^{1}$ Steward Observatory, University of Arizona, 933 North Cherry Avenue, Tucson, AZ 85721, USA \\ ${ }^{2}$ Observatories of the Carnegie Institution for Science, 813 Santa Barbara St, Pasadena, CA 91101, USA \\ ${ }^{3}$ Center for Astrophysics and Space Science, University of California San Diego, La Jolla, CA 92093, USA
}

Accepted 2013 May 27. Received 2013 May 17; in original form 2013 February 27

\begin{abstract}
We present low-resolution near-infrared (IR) 0.8-2.5 $\mu \mathrm{m}$ spectra of Supernova (SN) 2009ip, taken immediately before, during and just after its rapid brightening in late September/October 2012. The first epoch shows the same general spectral characteristics as the later epochs (smooth continuum, narrow $\mathrm{H}$ and $\mathrm{He}$ I emission lines), but the IR continuum shape is substantially redder than the later epochs. The epoch 1 continuum can be approximated by reddening the peak-luminosity (epoch 3 ) spectrum by $E(B-V)=1.0$ mag, but the blue colour seen in visual-wavelength spectra at the same time indicates that strong wavelength-dependent extinction by circumstellar dust is not the correct explanation. Instead, we favour the hypothesis that the redder colour before the brightening arises from excess emission from hot $\sim 2000 \mathrm{~K}$ circumstellar dust. The minimum radius $(\gtrsim 120 \mathrm{au})$ deduced from the dust temperature and observed luminosity of the transient, combined with the observed expansion speed in the precursor outbursts of SN 2009ip, is consistent with an ejection at least $1.1 \mathrm{yr}$ earlier. The mass of hot dust indicated by the IR excess is $\sim 4 \times 10^{-7} \mathrm{M}_{\odot}$, although this is only a lower limit since the near-IR data do not constrain the mass of cooler dust. Thus, the observed pre-SN outbursts of this object were able to efficiently form dust into which the SN ejecta and radiation now propagate. This is consistent with the notion that the same pre-SN eruptions that generally give rise to $\mathrm{SNe}$ IIn also give rise to the dust needed for their commonly observed IR echoes. We also discuss some aspects of the IR line profiles, including $\mathrm{He}_{\mathrm{I}} \lambda 10830$.
\end{abstract}

Key words: circumstellar matter-stars: massive-supernovae: general-supernovae: individual: SN 2009ip-stars: variables: S Doradus - stars: winds, outflows.

\section{INTRODUCTION}

Supernova (SN) 2009ip provided a truly remarkable sequence of events, first noted as an SN 'impostor' or eruption of a luminous blue variable (LBV) in 2009 (Smith et al. 2010), and then subsequently observed as a core-collapse SN in mid/late 2012 (Mauerhan et al. 2013). It joins SN 1987A (Rousseau et al. 1978; Walborn et al. 1989) as the only SN for which we have a direct detection and a spectrum of the progenitor star. In the case of SN 2009ip, however, we have a much more detailed record and higher quality data pertaining to its unstable pre-SN state. It is reminiscent of the pre-SN outburst seen $2 \mathrm{yr}$ before SN 2006jc (Pastorello et al. 2007), but in that case only one outburst was detected and no spectrum was obtained. A brief recounting of SN 2009ip's pre-SN activity is as follows.

After the discovery of SN 2009ip as a new transient source in 2009 August (Maza et al. 2009), it was recognized that it was not

\footnotetext{
^E-mail: nathans@as.arizona.edu
}

a true $\mathrm{SN}$, but was instead a non-terminal eruptive transient similar to LBVs. Smith et al. (2010) presented archival Hubble Space Telescope (HST) images, archival ground-based photometry, and new photometry and spectra of the outburst. HST data revealed that $10 \mathrm{yr}$ prior to discovery, the quiescent progenitor was a luminous supergiant with $L \simeq 10^{6} \mathrm{~L} \odot$ and a likely initial mass of around 50$80 \mathrm{M}_{\odot}$, while the archival ground-based photometry documented an $\sim 5$ yr long S Dor-like LBV phase that preceded the 2009 discovery. The 2009 outburst itself had a peak absolute magnitude of around -14.5 (Smith et al. 2010), similar to giant LBV eruptions (Smith et al. 2011). Spectra of the 2009 outburst showed strong Balmer emission lines similar to LBV eruptions (e.g. Smith et al. 2011), indicating an expansion speed of the order of $550 \mathrm{~km} \mathrm{~s}^{-1}$. The 2009 outburst of SN 2009ip was unusual compared to most LBV-like eruptions, however, due to the rapid brightening and fading over a few weeks instead of a few months to years (Smith et al. 2011). Foley et al. (2011) presented additional spectra and a reanalysis of the archival HST data that reinforced these conclusions. After these first papers on the 2009 outburst, SN 2009ip then had 
another outburst of similar magnitude in 2010 (Drake et al. 2010), and it has recently been reported (Pastorello et al. 2013) that it had additional eruptions of similar brightness since then as well.

On 2012 July 24 (UT dates are used throughout this paper), SN 2009ip was discovered to have yet another outburst (Drake et al. 2012). This new outburst was of similar magnitude to the previous ones, and at first it was assumed that this was yet another LBV-like eruption. Foley et al. (2012) reported that an early spectrum obtained on 2012 August 24 was consistent with the spectra of the previous LBV-like outbursts, with line widths around $600 \mathrm{~km} \mathrm{~s}^{-1}$. However, spectra obtained later on 2012 September 7-16 (Smith \& Mauerhan 2012) revealed a transformation in the spectrum of SN 2009ip, which now showed very broad Balmer emission and absorption lines out to speeds of $13000 \mathrm{~km} \mathrm{~s}^{-1}$. These broad features indicated that the impostor SN 2009ip was becoming a true core-collapse SN (Mauerhan et al. 2013), since these spectral features had never been seen previously in this object or any LBV eruption. Pastorello et al. (2013) have pointed out that broad features were seen previously, but these were only seen in absorption (as noted earlier by Smith et al. 2010; Foley et al. 2011), while emission-line components of SN 2009ip had always shown narrow or intermediate-width emission lines prior to 2012 September. Small amounts of very fast material have been seen around LBVs, as in the case of $\eta$ Carinae (Smith 2008), but the bulk velocity of most of the ejected mass in LBV eruptions is much slower.

The broad emission lines in SN 2009ip were first noted in 2012 September, around the time of peak luminosity for the precursor outburst (see Fig. 1). The object faded over the next week or so, but then brightened very suddenly, rising $\sim 3$ mag in a few days. This very rapid brightening was probably caused by the fast $\mathrm{SN}$ ejecta crashing into slower circumstellar material (CSM) ejected by the precursor eruptions (Mauerhan et al. 2013). Prieto et al.

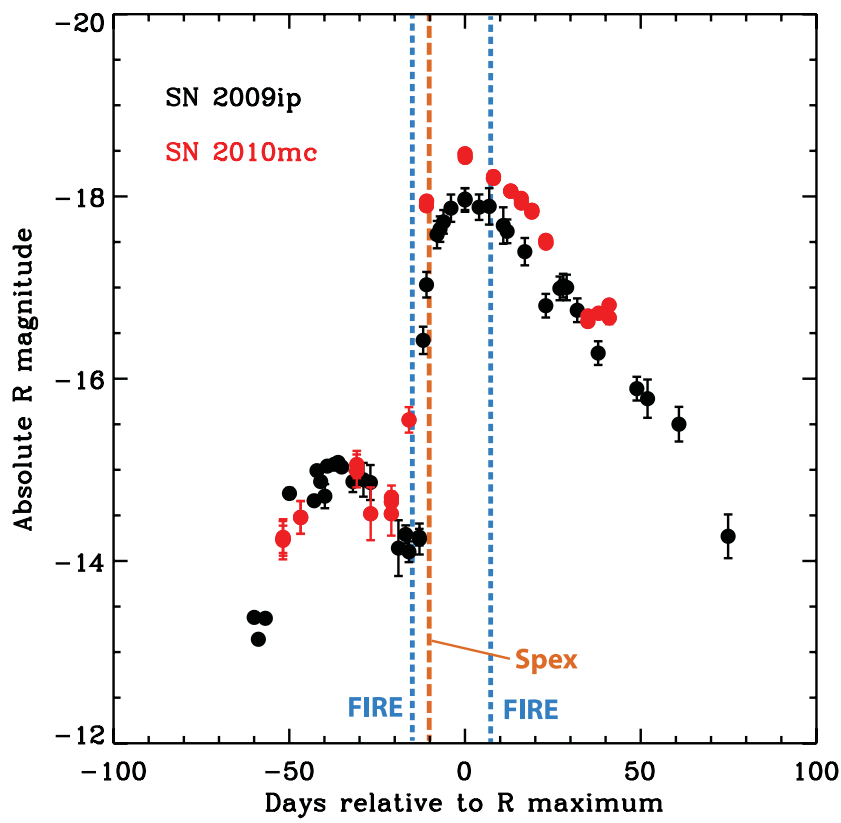

Figure 1. Absolute $R$-band magnitude light curve of SN 2009ip (black points) from Mauerhan et al. (2013; but with the three earliest points taken from Pastorello et al. 2013), showing the dates of our FIRE and SpeX spectra relative to the time of maximum luminosity (see Table 1 ). The red points show the recently published light curve of SN $2010 \mathrm{mc}$ from Ofek et al. (2013a), which appears to have an almost identical luminosity evolution (i.e. the absolute $R$-band magnitude of SN $2010 \mathrm{mc}$ was not scaled).
(2013) presented exceptionally high-cadence photometry of this very rapid brightening phase, also concluding that this was most likely due to the onset of SN-CSM interaction similar to other $\mathrm{SNe}$ IIn. Indeed, as it reached peak luminosity, the spectrum took on the character of a normal SN IIn with a smooth blue continuum and Balmer lines exhibiting narrow line cores and Lorentzian scattering wings (Mauerhan et al. 2013). Levesque et al. (2013) have recently presented additional spectra of the SN, highlighting aspects of its Balmer decrement. SN 2009ip gives vivid confirmation of the idea that very massive and eruptive LBV-like stars can explode to produce SNe IIn (e.g. Gal-Yam et al. 2007; Smith et al. 2007, 2008b, 2011; Gal-Yam \& Leonard 2009).

In Fig. 1 we also plot the light curve of SN 2010mc for comparison, recently reported by Ofek et al. (2013a). Ofek et al. discussed the remarkable detection of a precursor outburst in this Type IIn event as well, but they did not note the uncanny similarity to the light curve of SN 2009ip (which was still underway as their paper was in preparation). Fig. 1 emphasizes that these two objects are nearly identical in terms of peak luminosities and time-scales for both the precursor and $\mathrm{SN}$ outbursts (the absolute magnitudes have not been adjusted). Since most SNe IIn observed thus far have been discovered near peak and lack high-quality pre-SN observations, it may be the case that the erratic pre-SN variability of SN 2009ip is not so unusual.

Here we present near-infrared (IR) spectra of the remarkable 2012 explosion of SN 2009ip. We discuss implications for the dusty environment produced by the precursor LBV eruptions, as well as aspects of the line profiles.

\section{OBSERVATIONS}

We obtained three IR spectra of SN 2009ip, which were taken immediately before its fast rise in brightness, during the rise and around the time of peak luminosity. Fig. 1 shows the three epochs of our spectroscopy relative to the light curve of SN2009ip's 2012 outburst from Mauerhan et al. (2013). Observational information is listed in Table 1, and the resulting spectra are plotted in Fig. 2. All spectra in Fig. 2 (plotted in black) have been corrected for an assumed Galactic reddening value of $E(B-V)=0.019 \mathrm{mag}$, following Smith et al. (2010).

Our first near-IR spectrum was obtained on 2012 September 22 (UT) using the Folded-port InfraRed Echellette (FIRE) spectrograph (Simcoe et al. 2008, 2010) on the $6.5 \mathrm{~m}$ Magellan Baade Telescope. This was only $2 \mathrm{~d}$ prior to the dramatic rapid rise in brightness of SN 2009ip. The low-dispersion, high-throughput prism mode provides a spectrum that spans a wavelength range of $0.8-2.5 \mu \mathrm{m}$ at a resolving power $\lambda / \Delta \lambda=300-500$. We completed an ABBA sequence on SN 2009ip, with an average airmass of 1.16, and immediately afterwards obtained a spectrum of an A0 V standard star (HIP 113376) for flux calibration and telluric correction, as described by Vacca, Cushing \& Rayner (2003). Data were reduced using the FIREHOSE pipeline. Portions of this spectrum were presented and discussed by Ofek et al. (2013b) regarding relative line velocities.

Table 1. IR spectroscopy of SN 2009ip.

\begin{tabular}{lcccc}
\hline $\begin{array}{l}\text { Date } \\
(\mathrm{y}-\mathrm{m}-\mathrm{d})\end{array}$ & $\begin{array}{c}\text { Day } \\
(\max )\end{array}$ & Tel./instr. & $R$ & $\begin{array}{c}\Delta \lambda \\
(\mu \mathrm{m})\end{array}$ \\
\hline $2012-09-22$ & -15 & Magellan/FIRE & $300-500$ & $0.8-2.5$ \\
$2012-09-27$ & -10 & IRTF/SpeX & 120 & $0.7-2.5$ \\
$2012-10-14$ & +7 & Magellan/FIRE & $300-500$ & $0.8-2.5$ \\
\hline
\end{tabular}



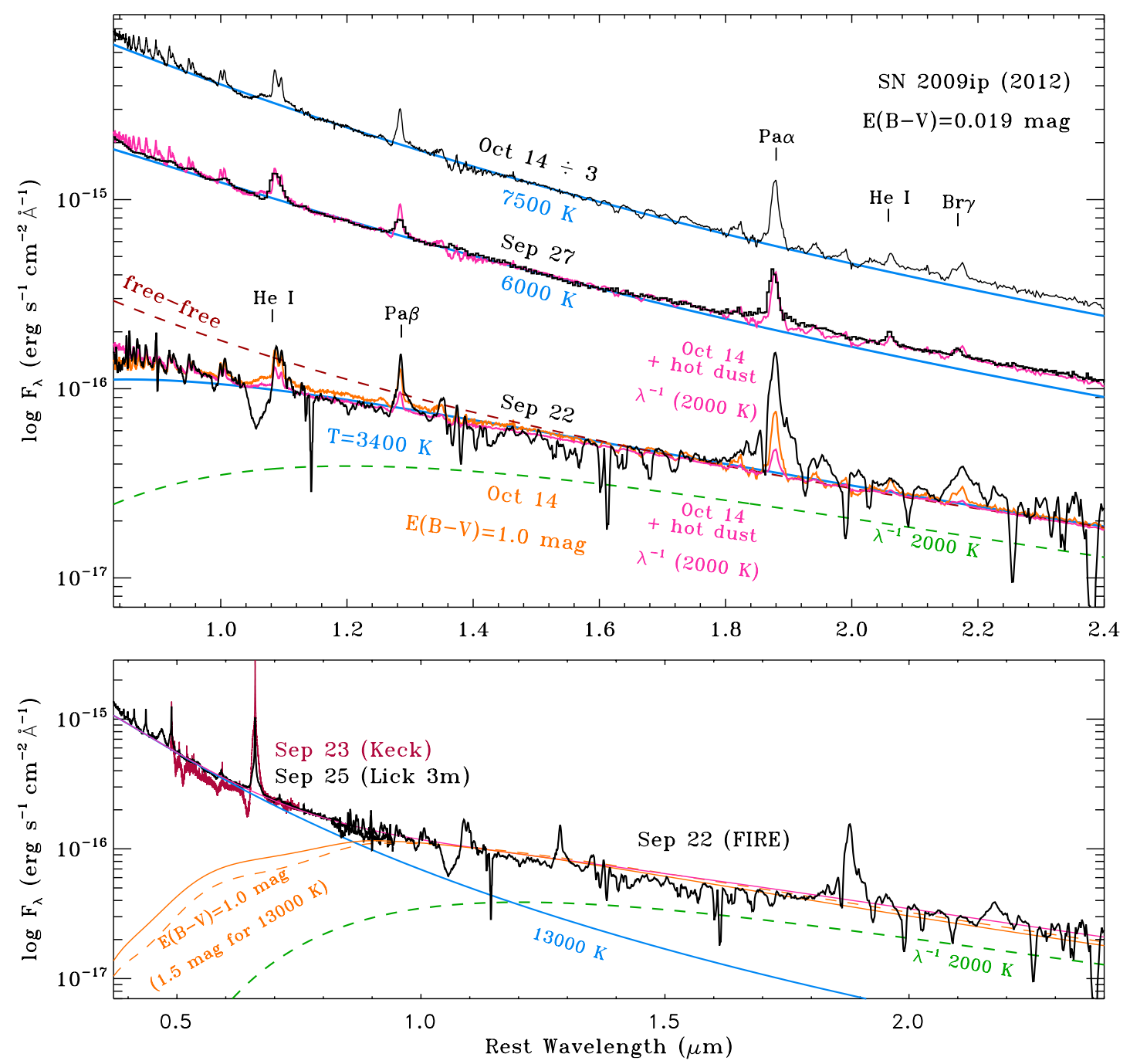

Figure 2. Top: near-IR spectra of SN 2009ip during the 2012 brightening. The black spectra are taken with SpeX and FIRE on the dates indicated (see Table 1), corrected for Galactic reddening of $E(B-V)=0.019$ mag (Smith et al. 2010). The coloured curves show various possible interpretations to match the continuum shape: (1) the blue curves show representative blackbodies; (2) the orange spectrum shows the October 14 spectrum (near peak luminosity) reddened by $E(B-V)=1.0 \mathrm{mag}$ to match the continuum shape of the pre-SN spectrum on September 22; (3) the two magenta spectra once again use the October 14 spectrum, but this time the continuum shape is augmented by a $2000 \mathrm{~K}$ greybody with $\lambda^{-1}$ emissivity, giving a rough example of how hot dust might add to the spectrum (the dust contribution is shown alone by the green dashed curve). The magenta spectrum matched to the September 27 spectrum has less relative contribution from hot dust, and relatively more from the scaled October 14 spectrum. Bottom: the same September 22 IR spectrum as in the top panel, but plotted alongside nearly simultaneous visual-wavelength spectra taken 1-3d later, from Mauerhan et al. (2013). The shape of the combined optical/IR continuum is best explained by a $13000 \mathrm{~K}$ blackbody (blue) plus the same $2000 \mathrm{~K}$ dust component as in the top panel (green dashed; the magenta curve shows the addition of the two components). Also shown for comparison are a $7500 \mathrm{~K}$ blackbody reddened by $E(B-V)=1.0 \mathrm{mag}$ (orange solid curve, as in the top panel) and a hotter $13000 \mathrm{~K}$ blackbody reddened by $1.5 \mathrm{mag}$ (orange dashed).

However, we noted that there may have been thin cirrus clouds during the observation of the standard star, which might affect the absolute flux calibration; because of this and possible slit losses that are difficult to quantify, the resulting flux-calibrated spectrum appeared brighter than indicated by simultaneous photometry, so the spectrum is scaled down in Fig. 2.

A second epoch of low-resolution near-IR spectra for SN 2009ip was obtained with the SpeX spectrograph (Cushing, Vacca \& Rayner 2004) on the NASA Infrared Telescope Facility (IRTF) on 2012 September 27 (UT) in clear conditions with 0.5 arcsec seeing. This spectrum was taken during the rapid rise, well before SN 2009ip had reached its peak luminosity, and an initial report was given by Burgasser, Nicholls \& Aberasturi (2012). The prismdispersed mode and 0.5 arcsec slit (aligned with parallactic) were used, providing $0.7-2.5 \mu \mathrm{m}$ coverage with resolution $\lambda / \Delta \lambda \approx 120$ and dispersion of 20-30 $\AA$ pixel ${ }^{-1}$. Eight exposures were obtained in an ABBA pattern at an average airmass of 1.52 , for a total exposure of $840 \mathrm{~s}$. The nearby A0 V star HD 202941 ( $V=7.05 \mathrm{mag})$ was observed immediately after for flux calibration and telluric absorption correction, along with internal flat-field and arc lamp exposures. Data were reduced with the IDL SPEXTOOL package, version 3.4 (Vacca et al. 2003; Cushing et al. 2004), using standard settings.

Our third epoch was obtained on 2012 October 14 (UT), once again using FIRE on Magellan. The low-resolution instrumental setup, observations of an $\mathrm{A} 0 \mathrm{~V}$ standard and data reduction procedures were nearly the same as described above for the previous FIRE epoch, except that we used a different standard star (HD 219341). 


\section{DUST AND THE IR CONTINUUM SHAPE}

A striking aspect of the spectral sequence in Fig. 2 (top) is that the first-epoch spectrum on September 22 is substantially different from those on September 27 and October 14, which appear qualitatively very similar to each other. One particular difference is in the continuum shape. For comparison, representative blackbody curves (blue) are plotted along with each epoch of the observed spectra, suggesting that the apparent colour temperature increases from $\sim 3400 \mathrm{~K}$ on September 22, to 6000 and $7500 \mathrm{~K}$ on the two later epochs. Continuum slopes matching temperatures of $6000-7500 \mathrm{~K}$ are typical for SNe IIn, and the spectral characteristics (strong $\mathrm{H}$ lines) are consistent with that range of temperatures. However, the much lower temperature of $3400 \mathrm{~K}$ implicated by the IR continuum slope on September 22 is not commensurate with the presence of $\mathrm{He}_{\mathrm{I}}$ and $\mathrm{H}$ emission lines in the spectrum. Therefore, a cooler photospheric temperature is not the likely explanation for the continuum shape (the $3400 \mathrm{~K}$ blackbody underpredicts the flux near $1 \mu \mathrm{m}$ anyway). Two possible alternatives involving dust are discussed below.

First, a redder colour on September 22 than at later times could potentially arise from significant reddening by CSM dust, which is destroyed at later epochs as the SN brightens and the shock expands. The orange curve in Fig. 2 (top panel) shows the October 14 spectrum reddened by $E(B-V)=1.0 \mathrm{mag}$, providing a satisfactory match to the shape of the observed spectrum on September 22. This is unlikely to be the real explanation for the observed colour, however, because the amount of reddening required to match the IR continuum shape would cause even more severe reddening at visual wavelengths. This is demonstrated in the bottom panel of Fig. 2, where we plot visual-wavelength spectra taken 1-3 d later (already published by Mauerhan et al. 2013) alongside the September 22 FIRE spectrum. The two orange curves (solid and dashed) show hot blackbodies (7500 and $13000 \mathrm{~K}$, respectively) that are reddened to approximate the shape of the near-IR continuum, using $E(B-$ $V)=1.0$ and $1.5 \mathrm{mag}$, respectively. While these reddened blackbodies seem to match the IR continuum shape, the flux at visual wavelengths falls far below the observed visual-wavelength spectra taken only 1 and $3 \mathrm{~d}$ later (note that these visual spectra have been adjusted in flux by a few per cent so that the $\sim 9000 \AA$ fluxes match the IR spectra, correcting for the fact that SN 2009ip was variable).

Instead of reddening, a more likely explanation for the IR colour is an extra contribution from emission of dust instead of absorption. The magenta spectrum plotted against the observed September 22 spectrum is, once again, the spectrum near peak on October 14, but with an added contribution from hot dust emission (Fig. 2, top panel). For simplicity, we used a $2000 \mathrm{~K}$ blackbody modified by $\lambda^{-1}$ emissivity. When added to the October 14 spectrum, it matches the overall shape of the September 22 near-IR continuum very well. The bottom panel of Fig. 2 shows that the combination (magenta curve) of a hot blackbody $(13000 \mathrm{~K}$, blue) and the same $2000 \mathrm{~K}$ dust component (green dashed) can match both the visualwavelength and IR spectra simultaneously. The fact that the visualwavelength continuum requires a hotter blackbody (13 000) than the temperature implied by fitting the IR continuum alone $(7500 \mathrm{~K})$ merely reflects the fact that IR wavelengths on the Rayleigh-Jeans tail of a Planck function are a less sensitive probe of the underlying hot component.

This IR excess presumably arises from CSM dust heated by the luminosity of the precursor transient source, as in an 'IR echo'. Dust at around this temperature is as hot as dust can be, residing at the innermost radius of the dust shell, inside of which the dust is destroyed. ${ }^{1}$ This general picture is similar to the IR echoes observed in many SNe, particularly common among SNe IIn (see Fox et al. 2011, and references therein) due to their dense and dusty preexisting CSM. On September 22 before the rapid brightening, SN 2009ip had a luminosity of roughly $L \simeq 4 \times 10^{7} \mathrm{~L}_{\odot}$. CSM dust in equilibrium at $2000 \mathrm{~K}$ would then reside at a radius of

$R \simeq 120 \mathrm{au}\left(\frac{Q L}{10^{7.6} \mathrm{~L}_{\odot}}\right)^{\frac{1}{2}}\left(\frac{T_{\mathrm{d}}}{2000 \mathrm{~K}}\right)^{-2}$

(where $Q$ is the ratio of the grain absorption to emission efficiency), which is around 120 au for large grains that behave like blackbodies ( $Q \simeq 1$ ), or somewhat farther from the star for smaller grain sizes that have larger values of $Q$. Equation (1) really provides a lower limit to the radius of the dust shell, since it could be farther away if dust is condensing as an ejected shell expands and cools (e.g. Kochanek et al. 2011). This is, therefore, probably not dust that formed from the beginning of the 2012 eruption $\sim 40 \mathrm{~d}$ earlier, because that material would be too close to the star. Expanding at around $550 \mathrm{~km} \mathrm{~s}^{-1}$ (Smith et al. 2010), the implied age is at least $1.1 \mathrm{yr}$, making it seem quite plausible that this dust formed from ejecta in one of its previous documented outbursts (see Drake et al. 2010; Smith et al. 2010; Mauerhan et al. 2013; Pastorello et al. 2013). ${ }^{2}$ In any case, it should be noted that this hot CSM dust that contributes to the emission in the IR spectrum on September 22 will not necessarily cause any corresponding absorption along our line of sight to the $\mathrm{SN}$ photosphere, since the geometry may be globally non-spherical or clumpy.

The contribution of this dust emission could weaken as time proceeds, either because some of the nearby CSM dust gets destroyed or because the dust emission simply makes a smaller contribution to the total flux as the brightness of the SN photosphere increases. ${ }^{3}$ Both appear to be happening. By the time SN 2009ip reached its peak, it had a luminosity of roughly $1.3 \times 10^{9} \mathrm{~L} \odot$. The same dust that was at $R=120$ au and $2000 \mathrm{~K}$ would then be heated to an equilibrium temperature of $4800 \mathrm{~K}$ (equation 1), vaporizing dust grains at that radius. (Moreover, the blast wave of the $\mathrm{SN}$ itself may have been approaching roughly the same radius by this point.) This destruction should happen as the SN brightens, but the IR excess might not disappear if there is additional dust at larger radii from previous outbursts that could be heated to the same temperature by the increasing luminosity. In fact, some IR excess emission does seem to persist. The magenta spectrum plotted against the September 27 spectrum in Fig. 2 (top) shows the same contribution of hot dust at the same $2000 \mathrm{~K}$ temperature, except that the relative contribution of the dust is less. This smaller relative contribution from hot dust matches the spectral shape very well, especially the excess flux longwards of $1.8 \mu \mathrm{m}$, and mimics the appearance of a cooler photospheric temperature $(6000 \mathrm{~K}$ versus $7500 \mathrm{~K}$ at peak).

\footnotetext{
${ }^{1}$ Note that a high dust temperature near $2000 \mathrm{~K}$ does not necessarily indicate $\mathrm{C}$-rich dust, as is often assumed. Dust formed in the colliding-wind shock of $\eta$ Carinae has a similar high temperature (Smith 2010) even though $\eta$ Car is known to be severely carbon deficient. For $\eta$ Car, the hot dust is thought to be corundum $\left(\mathrm{Al}_{2} \mathrm{O}_{3}\right)$, which condenses at a similar high temperature.

${ }^{2}$ In this context, it is interesting to note that Foley et al. (2011) reported a similar $\sim 2100 \mathrm{~K}$ dust excess around the LBV-like transient UGC 2773-OT. ${ }^{3}$ Note that our first spectrum was taken just $2 \mathrm{~d}$ before the rapid brightening, when the optical flux had faded by about $1 \mathrm{mag}$ from the peak of the precursor event (see Fig. 1). This drop in flux from the underlying photosphere would serve to enhance the contrast of the IR excess from dust at this epoch.
} 
A smaller contribution of hot dust may also be present even in the October 14 spectrum around the time of peak luminosity, since it appears to have a small amount of excess flux beyond $2 \mu \mathrm{m}$.

The mass of hot dust can be inferred from the IR excess if the grains are small $(a \lesssim 0.2 \mu \mathrm{m}$; following Smith \& Gehrz 2005; Smith, Foley \& Filippenko 2008a), which for grains with a density of $\rho=2.25 \mathrm{~g} \mathrm{~cm}^{-3}$ can be expressed as

$M_{\mathrm{d}} \approx 5 \times 10^{-7} \mathrm{M}_{\odot}\left(\frac{L_{\mathrm{d}}}{10^{7} \mathrm{~L}_{\odot}}\right)\left(\frac{T_{d}}{2000 \mathrm{~K}}\right)^{-6}$,

where $L_{\mathrm{d}}$ is the luminosity of the hot dust component at a temperature $T_{\mathrm{d}}$. The $2000 \mathrm{~K}$ dust that contributes the IR excess in our pre-SN spectrum on September 23 has a luminosity $L_{\mathrm{d}} \approx 9 \times 10^{6} \mathrm{~L}_{\odot}$, although this comes with a large uncertainty of perhaps \pm 50 per cent due to possible slit losses in the exposures of SN 2009ip or the standard star. Nevertheless, this provides a very rough estimate of the mass of hot dust of roughly (3-6) $\times 10^{-7} \mathbf{M}_{\odot}$. This is, of course, only a lower limit to the total circumstellar dust mass because our near-IR data only trace the hottest dust, while a large additional mass of cooler dust may emit at longer IR wavelengths.

Lastly, we note that additional IR flux from free-free emission due to an ionized stellar wind, which often causes IR excess in hot stars (Wright \& Barlow 1975), cannot explain the red colour because the slope from free-free emission is too blue at the shortest IR wavelengths. The dashed brown curve in Fig. 2 (top) shows the expected free-free IR emission from a constant-speed stellar wind.

\section{LINE PROFILES}

Line profiles for the three brightest lines in the IR spectrum are plotted in Fig. 3, showing 'before' and 'after' spectra of $\mathrm{He}$ I $\lambda 10$ 830, Pa $\beta$ and $\mathrm{Pa} \alpha$ taken at the same resolution with the FIRE spectrograph.

The most notable change in the line profiles is seen in $\mathrm{He}_{\mathrm{I}}$ $\lambda 10830$. In the first epoch before the brightening, this line exhibits a very strong and broad P Cygni absorption trough extending to a blue edge of $-13000 \mathrm{~km} \mathrm{~s}^{-1}$. This is the same speed as seen in the blue edge of the P Cygni absorption in Balmer lines over the preceding week or so (Mauerhan et al. 2013). This broad He I absorption then disappears when the $\mathrm{SN}$ brightens to peak. ${ }^{4}$ Balmer lines show the same disappearance of the broad profiles at peak (Mauerhan et al. 2013), presumably for the same reason - i.e. the spectrum becomes dominated by opaque CSM interaction. The narrow emission components of $\mathrm{He}$ I $\lambda 10830$ show little or no change before and after the rapid brightening, relative to the continuum. The metastable nature of $\mathrm{He}$ I $\lambda 10830$ absorption makes it difficult to use it to draw conclusions about the relative He abundance in the fast ejecta, and there are a few SNe IIn with comparable near-IR spectra for comparison.

$\mathrm{Pa} \beta$ and $\mathrm{Pa} \alpha$ do not show any strong broad $\mathrm{P}$ Cygni absorption, as is seen in Balmer lines and $\mathrm{He}_{\mathrm{I}} \lambda 10$ 830. There is a relatively faint underlying broad emission component to both Paschen lines, appearing as broad wings that weaken or disappear at the last epoch. The broad component makes a much stronger relative contribution to $\mathrm{Pa} \alpha$ than to $\mathrm{Pa} \beta$, which is the opposite of what is expected

${ }^{4}$ Note that some significant portion (20-50 per cent, depending on wavelength) of the near-IR continuum comes from hot CSM dust in an echo, rather than from the SN itself. This has the effect of diluting the apparent relative strength of emission/absorption lines. In other words, the IR emission/absorption features are stronger than they appear in this spectrum (i.e. the true intrinsic equivalent width is larger).

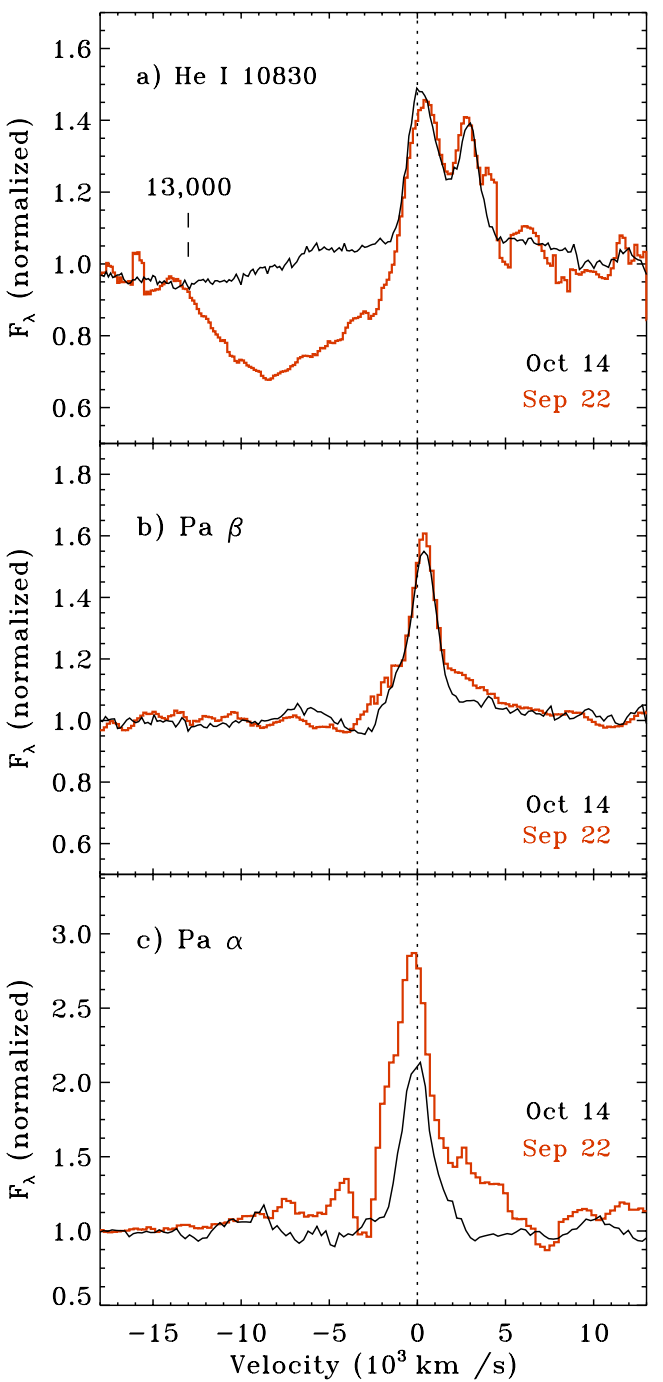

Figure 3. Individual line profiles before the sharp rise and soon after peak luminosity. (a) $\mathrm{He}$ I $\lambda 10$ 830, (b) $\mathrm{Pa} \beta$ and (c) $\mathrm{Pa} \alpha$.

for electron scattering, where higher order Paschen lines formed at higher optical depth have stronger electron scattering wings (Dessart et al. 2009). There is an apparent P Cygni absorption trough at roughly $-3000 \mathrm{~km} \mathrm{~s}^{-1}$ in $\mathrm{Pa} \alpha$ (with a width that is similar to the spectral resolution of our instrument), but it is difficult to judge the reality of this feature because of the strong telluric absorption in the vicinity of $\mathrm{Pa} \alpha$. As with $\mathrm{He}$ I $\lambda 10$ 830, the narrow components of the Paschen lines show little or no change with time, compared to the continuum, since they arise primarily in the CSM.

\section{ACKNOWLEDGEMENTS}

We thank an anonymous referee for helpful comments. MMK acknowledges generous support from the Hubble Fellowship and Carnegie-Princeton Fellowship.

Facilities: Magellan:Baade (FIRE), IRTF (SpeX).

\section{REFERENCES}

Burgasser A., Nicholls C., Aberasturi M., 2012, Astron. Telegram, 4431, 1 Cushing M. C., Vacca W. D., Rayner J. T., 2004, PASP, 116, 362 
Dessart L., Hillier D. J., Gezari S., Basa S., Matheson T., 2009, MNRAS, 394,21

Drake A. J. et al., 2010, Astron. Telegram, 2897, 1

Drake A. J. et al., 2012, Astron. Telegram, 4334, 1

Foley R. et al., 2011, ApJ, 732, 32

Foley R., Berger E., Roederer I., Chomiuk L., 2012, Astron. Telegram, 4338,1

Fox O. D. et al., 2011, ApJ, 741, 7

Gal-Yam A., Leonard D. C., 2009, Nat, 458, 865

Gal-Yam A. et al., 2007, ApJ, 656, 372

Kochanek C. et al., 2011, ApJ, 743, 73

Levesque E. et al., 2013, preprint

Mauerhan J. C. et al., 2013, MNRAS, 430, 1801

Maza J. et al., 2009, CBET, 1928, 1

Ofek E. O. et al., 2013a, Nat, 494, 65

Ofek E. O. et al., 2013b, ApJ, 763, 42

Pastorello A. et al., 2007, Nat, 447, 829

Pastorello A. et al., 2013, ApJ, 767, 1

Prieto J. L., Brimacombe J., Drake A. J., Howeton S., 2013, ApJ, 763, L27
Rousseau J. et al., 1978, A\&AS, 31, 243

Simcoe R. A. et al., 2008, Proc. SPIE, 7014, 27

Simcoe R. A. et al., 2010, Proc. SPIE, 7735, 38

Smith N., 2008, Nat, 455, 201

Smith N., 2010, MNRAS, 402, 145

Smith N., Gehrz R. D., 2005, AJ, 129, 969

Smith N., Mauerhan J., 2012, Astron. Telegram, 4412, 1

Smith N. et al., 2007, ApJ, 666, 1116

Smith N., Foley R. J., Filippenko A. V., 2008a, ApJ, 680, 568

Smith N. et al., 2008b, ApJ, 686, 467

Smith N. et al., 2010, AJ, 139, 1451

Smith N., Li W., Silverman J. M., Ganeshalingam M., Filippenko A. V., 2011, MNRAS, 415, 773

Vacca W. D., Cushing M. C., Rayner J. T., 2003, PASP, 115, 389

Walborn N. R. et al., 1989, A\&A, 219, 229

Wright A. E., Barlow M. J., 1975, MNRAS, 170, 41

This paper has been typeset from a $\mathrm{T}_{\mathrm{E}} \mathrm{X} / \mathrm{L} \mathrm{T}_{\mathrm{E}} \mathrm{X}$ file prepared by the author. 